\title{
Gata2a maintains cebpa and npm1a in haematopoietic stem cells to sustain lineage differentiation and genome stability
}

Christopher B. Mahony1,2, Boris Noyvert ${ }^{3}$, Pavle Vrljicak ${ }^{4}$, Sascha Ott ${ }^{4}$, Martin Higgs ${ }^{1,2,3}$, Rui Monteiro1,2,3,*

Addresses:

${ }^{1}$ Institute of Cancer and Genomic Sciences, College of Medical and Dental Sciences, University of Birmingham (UK), B15 2TT.

2 Birmingham Centre of Genome Biology, University of Birmingham, UK

${ }^{3}$ Cancer Research UK Birmingham Centre

${ }^{4}$ Division of Biomedical Sciences, Warwick Medical School, University of Warwick, Coventry, UK.

*corresponding author: r.monteiro@bham.ac.uk 


\section{Abstract}

The transcription factor Gata2 is required to produce and maintain haematopoietic stem and progenitor cells (HSPCs) in development and adult haematopoiesis. Mutations in GATA2 lead to GATA2 deficiency syndrome and predispose patients to acquire leukaemia. Here we use zebrafish gata2a enhancer deletion mutants and single cell transcriptomics to understand how GATA2 mediates survival and differentiation of haematopoietic stem cells in GATA2 deficiency. Gata2a mutants show marrow failure, neutropenia, B-lymphopenia and erythrocytosis from 6 months post-fertilization (mpf). Single cell transcriptional profiling of the adult kidney marrow demonstrated that HSPCs express elevated expression of erythroid- and decreased expression of myeloid genes, including cebpa. This is associated with a lineage skewing towards the erythroid fate at the expense of the myeloid fate. Thus, Gata2a is required to initiate and maintain lineage priming in HSPCs, favouring myeloid differentiation. Gata2a regulates expression of multiple targets associated with replication and DNA damage repair (DDR), including npm1a, a zebrafish NPM1 orthologue. Accordingly, mutant marrow cells show increased DNA damage associated with progressive loss of npm1a expression with age. This effect was replicated by inhibiting NPM1 activity in murine HPC7 progenitor cells. We propose that the impaired DDR leads to marrow failure in GATA2 deficiency. This leads to increased genomic instability in the surviving HSPCs, favouring acquisition of secondary leukaemogenic mutations. 


\section{Introduction}

The specification of haematopoietic stem and progenitor cells (HSPCs) from aortic endothelium during embryogenesis is a highly conserved process across many species. HSPCs mature and eventually seed their adult niche, the bone marrow in mammals and kidney marrow in zebrafish where they are able to self-renew, differentiate to any blood lineage and sustain haematopoiesis throughout life (Crane et al., 2017; Sawai et al., 2016). Lineage commitment and differentiation is tightly controlled by key genes and transcription factors.

GATA2 is a key haematopoietic transcription factor and is required for the formation and lineage output HSPCs and myelopoiesis (de Pater et al., 2013; Gao et al., 2013; Rodrigues et al., 2008). Loss of GATA2 expression due to GATA2 haploinsufficiency mutations in coding or enhancer regions causes haematopoietic disorders collectively referred to as GATA2 deficiency syndromes (Dickinson et al., 2014; Spinner et al., 2014). These patients are predisposed to mycobacterial or viral infections, suffer from lymphedema and develop marrow failure (McReynolds et al., 2018). The majority of GATA2 deficiency patients with inherited germline GATA2 mutations ( 75\%) develop early onset myelodisplastic syndrome (MDS) and Acute Myeloid Leukaemia (AML) at a median age of 20(Hirabayashi et al., 2017; Wlodarski et al., 2017). Existing mouse models fail to mimic the disease progression observed in humans; they either show no haploinsufficiency phenotype, are embryonic lethal or require exogenous stress to reveal haematopoietic repopulation defects (Gao et al., 2013; Johnson et al., 2012; Soukup et al., 2019). In contrast, by deleting a conserved intronic endothelial enhancer in the zebrafish gata2a locus (gata2a $a^{-/}$mutants hereafter), we generated a model of GATA2 deficiency (Dobrzycki et al., 2020) that show marrow hypocellularity, neutropenia and propensity 
to infections and to develop an AML-like phenotype in the adult kidney marrow (Dobrzycki et al., 2020). Here we make use of this model to investigate disease onset and study the transcriptional changes that occur at the single cell level in mutant haematopoietic cells to understand how gata2a deficiency leads to perturbed haematopoiesis, marrow failure and predisposition to MDS/AML.

We demonstrate that gata2a $/$ mutants show marrow hypocellularity from 6 months post-fertilization (mpf), accompanied by neutropenia, monocytopenia and increased numbers of erythrocytes. Single cell transcriptional profiling of whole kidney marrow (WKM, equivalent to bone marrow in humans) enabled us to identify all the major haematopoietic populations in the WKM and demonstrated that the neutropenia and erythrocytosis phenotypes are detectable at a molecular level at $5 \mathrm{mpf}$, prior to the marrow failure and cytopenia phenotypes. Gata2a $\%$ mutant HSPCs are mis-programmed, expressing increased levels of erythroid lineage genes (hemgn, igfbp2a, hbba1, hbaa1) and decreased expression of myeloid genes, including lcp1, npm1a and cebpa. Pseudotime analysis showed that loss of cebpa in gata2a $a^{\%}$ HSPCs correlated with increased expression of erythroid lineage genes, suggesting that cebpa is a downstream effector of gata2a in HSPCs. In addition, mutant HSPCs showed decreased expression of key replication and DNA damage repair genes, including $n p m 1 a$, indicating a possible mechanism for DNA damage repair in the bone marrow failure phenotype in GATA2 deficiency patients. Indeed, we show that npm1a expression is lost as disease progresses and correlates with the appearance of DNA damage in WKM and murine HPC7 multipotent progenitor cells. Taken together, gata2a is required to initiate and maintain lineage priming in HSPCs through cebpa, favouring the myeloid fate over the erythroid or B-lymphoid fates. Furthermore, we propose that gata2a regulates HSPC survival and protects against bone marrow failure by regulating expression of essential DNA damage 
repair genes. In the absence of gata2a, the impaired DNA damage repair machinery leads to increased DNA damage and decreased HSPC survival.

\section{Results}

\section{Gata2a directs myeloid lineage differentiation and maintains homeostasis in the} WKM

Our previous study of zebrafish gata2a revealed that gata2a enhancer (i4 enhancer) mutants show impaired haemogenic endothelium (HE) programming but HSPC numbers in the embryonic niche recover by 5dpf (Dobrzycki et al., 2020). However, at 6 months post-fertilization (mpf), adults exhibit a hypocellular WKM, neutropenia, and by $9 \mathrm{mpf} 1$ in 10 show $>98 \%$ blasts in the WKM, a hallmark of AML that indicated that the enhancer deletion mutants displayed key characteristics of GATA2 deficiency in humans (Dobrzycki et al., 2020). To uncover the mechanism underlying disease progression in GATA2 deficiency, we first determined the onset of the hypocellularity phenotype by analysing WKM from 4, 5 and 6mpf gata2a mutants by WKM smears and cell counts (Fig. 1A,B). These experiments revealed that the marrow hypocellularity only became apparent by $6 \mathrm{mpf}$ (Fig. $1 \mathrm{~A}, \mathrm{~B})$. However, the hypocellular phenotype was detectable in gata $2 a^{+/-}$heterozygous and was indistinguishable from homozygous mutants, indicating that maintenance of normal WKM cell numbers is very sensitive to the dose of gata2a (Fig. 1A, B). To examine how HSPC differentiation was affected, gata $2 a^{-/}$mutants were crossed to transgenic lines to examine haematopoietic populations in the WKM as previously established (Traver et al., 2003). We looked at neutrophils (mpx:GFP, myeloid gate) (Renshaw et al., 2006), macrophages (mpeg1.1:GFP, myeloid gate), B cell 
(mpeg1.1:GFP, lymphoid gate) (Ferrero et al., 2020) and erythrocytes (gata1:DsRed, erythroid gate) (Yaqoob et al., 2009) at 6mpf (Fig. 1C). As expected, we found decreased numbers of $m p x: G F P^{+}$neutrophils in gata $2 a^{\%}$ (Fig. 1D). In addition, we found decreased mpeg1.1:GFP+ B cells in the lymphoid gate (Fig. 1F) and increased gata1a:DsRed ${ }^{+}$cells in the erythroid gate (Fig. 1G). Together, these experiments showed that Gata2a is required to maintain haematopoietic cell numbers in the WKM and for correct lineage differentiation in the adult marrow. No significant skewing in lineage differentiation was found in gata2a heterozygous mutants, indicating that maintaining cellular homeostasis in the WKM requires higher levels of Gata2a and is more sensitive to Gata2a dosage than lineage determination.

\section{Single cell RNAseq analysis revealed lineage skewing towards the erythroid fate at}

\section{the expense of myeloid cell fates}

To investigate how gata2a regulates WKM cellularity and lineage differentiation, we undertook transcriptional profiling of the WKM at single cell resolution at 5mpf, prior to the onset of the detectable lineage skewing and hypocellularity phenotypes by flow cytometry. We took two adult fish per genotype, dissected and pooled the WKM and sorted live cells for scRNAseq using the 10X Chromium platform. Analysis estimated 3197 WT cells and 5838 gata2a $\%$ - cells. We could readily identify cell clusters representing all the major haematopoietic and kidney cell populations present in the WKM (Fig. 2A), consistent with recent studies (Moore et al., 2016; Tang et al., 2017). We defined 'core HSPCs' (cHSPCs) by expression of myb, Imo2, cebpa, meis1b, gata2b and runx1 (Fig. S1A, 2B). They were also enriched in other known embryonic HSPC markers such as gfi1aa, dnmt3ba, angpt1, and si:dkey-261h17.1 (identified as the zebrafish orthologue of CD34, 
zfin.org, hereafter referred to as cd34-like, or $c d 34 l$ ) (Fig. S1A, 2B). Phylogeny and synteny analyses revealed the conservation of the CD34 antigen as defined by ENSEMBL (Fig. S2A-C). We also defined a second HSPC population as 'myeloid-primed' HSPCs (mHSPCs) based on their lack of expression of pluripotency markers (such as gata2b, runx1, and meis $1 b$ ) and their retention of myeloid-biased transcription factors (Imo2, cebpa and myb) and higher expression levels of c1qa and mafba (Fig. S1A). We then defined the remaining populations based on their expression of key marker genes (igfbp1a, hemgn, klf1: Erythroid progenitors (Ery-P), c1qa, mfap4, mafba: monocytes/macrophages, $m p x, m m p 13 a$, vamp8: neutrophils, $n k l .3$, il2rb: NK/Tcells, pax5, cd37, cd79b: B cells, sox8b, tekt1: multi-ciliated kidney cells, spink2.2, viml: kidney tubule cells) (Fig. 2B, S1A). When we compared wildtype and gata2a $/$ - WKM we found a striking increase in erythroid cells and a decrease in neutrophils (Fig. 2C), indicating that the lineage skewing observed at $6 \mathrm{mpf}$ (Fig. 1) could be detected at the cellular and molecular level at $5 \mathrm{mpf}$ (Fig. 2) by single cell RNAseq.

When then examined differential gene expression in neutrophils, monocytes/macrophages, erythrocytes, NK/Tcells and B cells. Within the Ery-P we found no differentially expressed genes. Surprisingly, despite their increase in number, relatively few genes were significantly changed in erythrocytes (Fig 2D). They showed a decrease in $d r l l .1$, a transcription factor required for erythropoiesis (Kobayashi et al., 2020) and ectopic expression of aqp12 and $d h r s 4$, suggesting that although their number was increased, their function was likely impaired (Fig. 2D). Monocytes showed a decrease in the myeloid markers lcp1,f5 and f11r.1 (AKA jam-a) (Luissint et al., 2019) in gata2a $/$ cells indicating altered differentiation and function (Fig. 2D). gata2a $a^{-}$mutant neutrophils also expressed less lcp1 and f5 and more si:ch211-5k11.8 (Orthologous to human HBZ-haemoglobin zeta, zfin.org) and hbba1 (Fig. 2E). Mutant B cells expressed 
less Icp1 and more hbba1, hbaa1, hbba2, hbaa2 and si:ch211-5k11.8 (Fig. 2F). Mutant NK/T cells expressed more hbba1, hbaa1 and si:ch211-5k11.8 (Fig. 2E). In summary, the myeloid and lymphoid haematopoietic populations show downregulation of genes of myeloid lineage and ectopic upregulation of genes of the erythroid lineage. Taken together, this suggested that Gata2a, mainly expressed in the cHSPC population (Fig. 2A, $\mathrm{S} 1 \mathrm{~B})$, is required to maintain transcriptional states of myeloid and erythroid genes in the most differentiated haematopoietic populations and that aberrant gene expression in differentiated blood lineages could be traced back to the HSPC populations. To investigate this, we next examined differential gene expression in cHSPCs and mHSPCs (Fig. 3). Mutant cHSPCs showed a decrease in drll.1, as well as atp6V0e1 and mhc1uba, and an increase in the HBZ orthologue, si:ch211-5k11.8 (Fig. 3A). GO term enrichment analysis highlighted only very few pathways, suggesting that cHSPCs remain relatively unaffected (Fig.3C, D). Many more genes were differentially expressed in mHSPCs (Fig. 3B). We found that globin genes (e.g.hbba1, hbaa1, hbba2, hbaa2: Figure 3G), as well as known erythroid regulators like hemgn (Peters et al., 2018), igfbp1a, znlf2a and rhag, were all upregulated in mHSPCs, which was confirmed by GO term enrichment analysis which highlighted that upregulated genes were enriched in pathways for erythrocyte differentiation (Fig. 3E). By contrast, cebpa (Figure 3G) and myeloid lineage markers like lcp1, Isp1b and $f 5$ (Fig. 3B) were downregulated. cebpa is a key regulator of granulocyte and monocyte differentiation and primes HSPCs towards the myeloid fate (Avellino and Delwel, 2017; Dai et al., 2016; Hasemann et al., 2014; Suh et al., 2006). Thus, the differentiation skewing towards the erythroid lineage at the expense of myeloid lineages

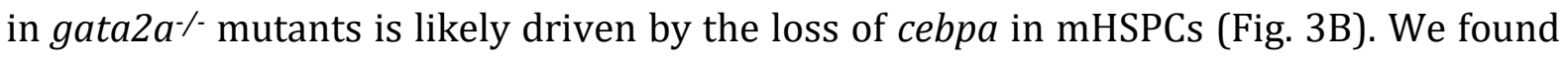
that critical regulators of DNA replication (e.g. $m c m 3, m c m 5$ : Fig. 3G) were downregulated in mHSPCs, suggesting a decrease in proliferation (Shinya et al., 2014; Snyder et al., 2005) 
consistent with the cytopenic phenotype noted at $6 \mathrm{mpf}$. In addition we noted a decrease in genes required for DNA repair and genome stability, such as hells (Kollárovič et al., 2020), npm1a (Grisendi et al., 2005; Koike et al., 2010) and ncl (Kawamura et al., 2019; Scott and Oeffinger, 2016) (Fig. 3G). This was confirmed by GO term enrichment analysis which highlighted that downregulated genes were enriched for pathways in DNA replication initiation, chromatin assembly/disassembly and methylation (Fig. 3F). Other GO terms included ribosome biogenesis, highlighted due to the previous role of NPM1 in ribosome biogenesis (Lindström, 2011), centromere formation and histone chaperoning (Grisendi et al., 2005; Okuwaki et al., 2001).

We conclude that gata2a expression in cHSPCs is required to establish a transcriptional network that controls erythroid and myeloid fate through cebpa, HSPC fitness and survival via regulation of metabolic pathways and the expression of DNA repair genes.

\section{Pseudotime analysis shows that lineage skewing in gata2a ${ }^{-/}$originates from HSPCs and correlates with loss of cebpa expression}

We noted that a number of genes were consistently up- (e.g. ier $2 a$, junbb) or downregulated (e.g. atp6V0e1, mhc1uba) across several haematopoietic populations including HSPCs (Fig. S2, Fig. 2, Fig. 3). To understand the dynamics of gene expression changes found in gata $2 a^{\%}$ mutants, we examined gene expression during HSPC differentiation using pseudotime analysis with Monocle 3 (Cao et al., 2019; Qiu et al., 2017a; Qiu et al., 2017b; Trapnell et al., 2014). For that, we first plotted the WKM populations using UMAP clustering and identified HSPCs based on their expression of Imo2, myb, gata2b, cd34l, meis1b and cebpa (Fig. S3A, B). We repeated the UMAP clustering in Monocle 3 and determined the expression of the same genes (Fig. S3C), 
which allowed us to select a broad HSPCs population (Fig. S3D) for further pseudotime analysis. We then reduced dimension and defined five distinct HSPC populations based on marker expression (Fig. 4A). We first defined cHSPCs based on their higher expression of $c d 34 l$, meis $1 b$, gata $2 b$ and Imo2 (Figure S4A). mHSPCs were defined by higher expression of cebpa, myb, and $c 1 q b p$ along with more restricted spi1b expression (Fig. S4B). Proliferating HSPCs were defined by high expression of histone genes (Fig. S4C). One population was enriched in haemoglobins which we classified as erythrocyte biased HSPCs (eryHSPCs, Fig. S4D). A fifth, small population of HSPCs was enriched in lymphoid genes (rag1, rag2, cxcr4b, btg1) and thus defined as lymphoid-biased HSPCs (lymphHSPCs, Fig. S4E). We then examined expression of key myeloid and erythroid genes in pseudotime by classifying cHSPCs as the differentiation origin. For this analysis, we excluded proliferating HSPCs. To understand how the expression of myeloid and erythroid genes changes with differentiation, we visualised the expression of cebpa, hbba2, hbaa2 and hemgn in pseudotime. We found that wild type cHSPCs and mHSPCs express cebpa at similar levels whereas it is downregulated as they differentiate towards eryHSPCs (Fig. 3B). By contrast, gata2a ${ }^{-}$cHSPCs and mHSPCs show severely decreased cebpa expression that is downregulated early on in pseudotime. The erythroid genes hbba2, hbaa2 and hemgn are expressed at low levels in wildtype cHSPCs and mHSPCs and are upregulated as HSPC differentiate to eryHSPCs. Both hbba2 and hbaa2 are upregulated in gata $2 a^{\circ}$ cHSPCs and robustly upregulated in mHSPCs and eryHSPCs (Fig. 3B). These results show that loss of cebpa as HSPCs differentiate correlated with enhanced erythroid marker expression and reduced myeloid marker expression, suggesting that a Gata2a-Cebpa axis mediates myeloid lineage priming in HSPCs. 


\section{The nucleophosmin orthologue npm1a is highly enriched in HSPCs}

Mutations in the C-terminal nuclear localization sequence of nucleophosmin (NPM1) are associated with myeloid and lymphoid malignancies (Sportoletti et al., 2008) and are found in around 30\% of AML cases (Falini et al., 2005). Furthermore NPM1 is associated with maintenance of genome stability and required for DNA repair (Grisendi et al., 2005; Koike et al., 2010). At 5mpf, expression of the NPM1 orthologue, npm1a expression was most highly enriched in cHSPCs and mHSPCs, along with $c d 341$ (Fig. S5A, A'). npm1a expression was decreased in gata2a $\%$ mHSPCs (Fig. 3B,G) at 5mpf, suggesting that it may play a role in disease progression in GATA2 deficiency. To investigate this, we first asked whether npm1a expression was detectable in HSPCs isolated from WKM by expression of low levels of GFP driven by the CD41 promoter (CD41:GFPlow HSPCs) (Ma et al., 2011). Because our experiment was performed using wildtype and gata2a/- WKM on a $c d 41: G F P$ transgenic background, we mapped $g f p$ expression and confirmed that it was highly enriched in mHSPCs and cHSPCs (Fig. S5A, A'). We grouped cHSPCs and mHSPCs into HSPCs and partitioned the resulting population into three groups: GFPnegative (expression less than $0.2(\log 2$ scale)), GFPlow (expression from 0.2 to 4 (log2 scale)) and GFPhigh (expression > 4 (log2 scale)). We then identified the global markers associated with each population compared to the WKM. The GFPnegative HSPCs displayed enrichment in erythroid markers (znfl2a, mycb, hbae1.3) and npm1a (Fig. S5B). As expected, the GFPlow population was highly enriched in HSPC-associated markers, including gata2b, cd34l, runx1t1 and npm1a. The GFPhigh population expressed higher levels of thrombocyte-associated genes including $m p l$ and $g p 1 b b$ (Figure S5D, E) but lacked npm1 $a$ and $c d 34 l$. This confirmed that npm1a is expressed in the CD41:GFPlow HSPC population and allowed us to examine whether loss of npm1a correlates with disease progression. 
We took two cd41:GFP and two cd41:GFP;gata2a $\%$ adults at $7 \mathrm{mpf}$, dissected WKM and sorted live, CD41:GFPlow cells from the lymphoid and progenitor gates (Fig. 5A). FACS analysis showed a decrease in the number of CD41:GFPlow cells in the mutant samples, as expected (Fig. 5A). We then processed the sorted cells for scRNA-Seq. Analysis estimated 700 WT cells (median UMI counts per cell=341, mean reads per cell $=123905$, median genes per cell $=156)$ and 1117 gata2a $\%$ cells (median UMI counts per cell=258, mean reads per cell $=75715$, median genes per cell $=128$ ). Due to low fraction reads, we chose cells with at least 1000 UMI counts in R and then exported extracted barcoded reads to be retained for further analysis in cell ranger/Loupe. We then analysed 106 wildtype cells (pooled from both samples) and 72 mutant cells (pooled from both samples) that revealed two distinctly separated GFPlow HSPC populations between wildtype and gata2a $/-$ HSPCs (Fig. 5B). tSNE plot of the cells showed two distinct clusters made up exclusively of either WT or gata2a $\%$ cells (Fig. 5C). Differential expression analysis showed a strong down regulation of $n p m 1 a$ in gata $2 a^{-}$HSPCs (Fig. 5D, E) and upregulation of innate immune response genes (lyz and lect2l, Fig. 5D, F: a likely consequence of a shift to myeloid blast accumulation preceding MDS/AML onset). Consistent with the erythroid skewing found in $5 \mathrm{mpf}$ WKM, hbba2 and hbaa2 were still upregulated in gata2a $/-$ HSPCs at 7mpf (Fig. 5D, F). Thus, we concluded that disease progression correlated with a decrease in npm1a expression in HSPCs.

\section{Loss of npm1a correlates with DNA damage}

Loss of Npm1 function in a murine hypomorphic mutant leads to genomic instability, impaired repair of DNA double strand break damage and promotes oncogenesis (Andrade et al., 2020; Grisendi et al., 2005). Thus, we wanted to examine if the decrease in $n p m 1 a$ in HSPCs correlates with increased DNA damage in the gata $2 a \%$ mutant WKM. 
For this, we examined $\gamma \mathrm{H} 2 \mathrm{AX}$ staining, a marker of DNA damage (Sharma et al., 2012) , in WKM smears at 6 and $12 \mathrm{mpf}$ (Fig. 6). As a positive control, irradiation with 10Gy induced nuclear $\gamma \mathrm{H} 2 \mathrm{AX}$ staining in a freshly isolated wildtype WKM smear (Fig. 6A). Wildtype WKM cells at 6 and $12 \mathrm{mpf}$ showed no evidence of $\gamma \mathrm{H} 2 \mathrm{AX}$ staining (Fig. 6B). By contrast, we could readily detect $\gamma \mathrm{H}_{2} \mathrm{AX}^{+}$cells in WKM from gata2a $\%$ adults at 6 and $12 \mathrm{mpf}$ (Fig. $6 \mathrm{~B}, \mathrm{~S} 6 \mathrm{~A})$. Flow cytometry analysis at $12 \mathrm{mpf}$ showed that increased $\gamma \mathrm{H} 2 \mathrm{AX}$ was also detected in gata2a $\%$ WKM suspensions suggesting that loss of gata2a leads to increased DNA damage in HSPCs (Fig. 6C). To independently confirm these results in a different model, we examined $\gamma \mathrm{H} 2 \mathrm{AX}$ following NPM1 inhibition in murine haematopoietic precursor HPC7 cells (Pinto do et al., 1998). Inhibition with $1 \mu \mathrm{M}$ of the NPM1 inhibitor for $24 \mathrm{~h}$ showed an increase in $\gamma \mathrm{H} 2 \mathrm{AX}$ (Figure 6D), indicating that NPM1/npm1a is required to prevent increased DNA damage and maintain genomic stability in HSPCs.

In summary, we concluded that gata2a regulates cebpa and npm1a in HSPCs. Loss of cebpa results in skewed differentiation towards erythroid lineage at the expense of the myeloid lineages (neutrophils and monocytes), whereas loss of npm1a results in the inability to efficiently repair DNA damage in HSPCs. We propose that this results in increased genomic instability leading to the accumulation of somatic mutations and disease progression from a first phase of hypocellular marrow to a second phase of myeloid proliferation in HSPCs and onset of MDS/AML in GATA2 deficiency.

\section{Discussion}

We have shown that deletion of a conserved gata2a enhancer (the i4 enhancer) leads to hypocellular marrow and a clear disease phenotype from $6 \mathrm{mpf}$. Loss of the i4 enhancer 
leads to ectopic expression of erythrocyte genes in HSPCs and myeloid and lymphoid lineages and differentiation skewing from $5 \mathrm{mpf}$ as a result of decreased cebpa expression. Furthermore, decreased npm1a expression in mHSPCs correlates with the appearance of DNA damage in WKM cells and in murine haematopoietic progenitor cells, HPC7. These transcriptional changes lead to aberrant differentiation and defective DNA repair that ultimately trigger the marrow failure phenotype.

In our scRNA-Seq, we find less cebpa in gata2a/- mHSPCs, despite gata2a being mainly expressed in cHSPCs. Genetic and epigenetic analysis of CEBPa indicates that it acts as an HSC priming factor to promote myeloid differentiation and protects HSCs against apoptosis and maintains quiescence (Hasemann et al., 2014; Suh et al., 2006). We therefore hypothesise that gata2a sets up a transcriptional network in cHSPCs, possibly through chromatin remodelling, that is required for the expression of cebpa in mHSPCs. Furthermore, a downstream CEBPa enhancer is bound by GATA2 (Cooper et al., 2015), suggesting that the equivalent cebpa enhancer could be directly regulated by gata2a in zebrafish HSPCs. We therefore conclude that in our gata2 $a^{-/-}$mutants, as cHSPCs begin to differentiate to mHSPC, lack the epigenetic cues that maintain cebpa expression and therefore differentiate to more erythroid lineages at the expense of myeloid ones (Suh et al., 2006). This is illustrated well by our pseudotime analysis of HSPC differentiation (Fig. 4) where cebpa expression is much lower in the gata2a $a^{-/-}$mutants and erythroid genes are ectopically expressed.

Analysis of the gata2a zebrafish orthologue, gata2b, found that it was responsible for myeloid lineage commitment, over lymphoid and erythroid, when examining the transcriptional changes in immature gata2b deficient HSPCs (Gioacchino et al., 2021). Further analysis showed alterations in chromatin accessibility in early myeloid and 
lymphoid progenitors from whole marrow studies in a different gata $2 b$ mutant leading to defects in myeloid and lymphoid differentiation (Avagyan et al., 2021). As both gata2a and gata $2 b$ are expressed in HSPCs and that loss of either gene leads to a defect in myelopoiesis/lymphopoiesis we conclude that they have overlapping roles in HSPC biology and would function as "allelic paralogues" to recapitulate the human phenotype in germline heterozygous LOF mutations. However, our gata2a mutant (Dobrzycki et al., 2020) recapitulates a wider range of GATA2 dficiency syndrome symptoms including the recurrent infections, lymphedema, cytopenia and predisposition to MDS/AML and thus more faithfully recapitulates GATA2 deficiency syndrome.

In our characterisation of the WKM cell population by scRNA-Seq we identified the zebrafish orthologue of cd34 (cd34l/ si:dkey-261h17.1). Interestingly, the function of CD34 is to enable HSPCs to anchor to their marrow niche (Healy et al., 1995). We noted that cd34l was also modestly decreased in mHSPCs (Fig. 3B), which is likely to be contributing to the hypocellular phenotype seen in our model. We hypothesize that with less $c d 341$, HSPCs anchor themselves to the WKM niche less efficiently (Healy et al., 1995) and are no longer exposed to supportive niche signals and therefore differentiate and reduce their proliferation.

Appearance of MDS/AML in patients carrying germline GATA2 mutations is associated with secondary mutations in several genes (Hirabayashi et al., 2017; McReynolds et al., 2019; Wang et al., 2015; West et al., 2014). Loss of one Gata2 allele in a murine CbfbMYH11 fusion AML model leads to a 2-fold increase in somatic mutations (Saida et al., 2020). While these studies provide an association between the genotype (mutated genes) and the phenotype (leukaemia), they fail to address how the identified mutations arise or how they affect gene expression to initiate or exacerbate disease progression. 
Here, we have found that loss of gata2a leads to less npm1a and the acquisition of DNA damage. Although it remains unknown whether the regulation of npm1a by gata2a is direct, previous studies have shown that NPM1 maintains genome integrity during cell cycle, inhibits apoptosis/tumour suppression through p53 and its loss of function is associated with myeloid and lymphoid malignancies (Colombo et al., 2002; Okuwaki, 2008; Sportoletti et al., 2008). We therefore conclude that loss of npm1a would lead to increased genomic instability and acquisition of secondary mutation over time. Once HSPC clones acquire sufficient combinations of pathogenic mutations in hematopoietic genes, they are conferred a competitive advantage that permits their clonal expansion and would eventually trigger MDS/AML. The acquisition of secondary mutation may correlate with the appearance of disease noted at $6 \mathrm{mpf}$ in this study and would be interesting to follow up with further single cell multi-omics studies to investigate the pathways dysregulated downstream of specific mutation combinations. This will pave the way to undertake drug screening studies and develop more personalized treatments to help treat marrow failure and disease progression to MDS/AML.

\section{Materials and Methods}

\section{Zebrafish}

$\mathrm{AB}^{*}$ zebrafish strains, along with transgenic strains and mutant strains, were kept in a $14 / 10 \mathrm{~h}$ light/dark cycle at $28^{\circ} \mathrm{C}$. We used the following transgenic animals: $c d 41$ :GFP (Lin et al., 2005), Tg(flk1:Hsa.HRAS-mCherry)s896 (Chi et al., 2008), mpx:GFPc264Tg (Renshaw et al., 2006), mpeg1:GFPgl22 (Ellett et al., 2011). We used the following mutant animals: gata2a (Dobrzycki et al., 2020). Experimental procedures in adult fish were 
done in accordance with the Animal Scientific Procedure Act 1986 under an approved Home Office Project License (PPL2470547).

\section{May-Grundwald (MG) and Giemsa staining WKM smears}

Whole kidney marrow tissue was dissected from sacrificed animals and smeared on a superfrost plus slide (Thermo Scientific) and left to air dry for at least $1 \mathrm{~h}$ at RT. MG (Sigma) was diluted 1:1 and Giemsa (Sigma) 1:10 with distilled water. Slides were incubated with diluted MG for 5 min at RT, then washed and repeated with diluted Giemsa for $30 \mathrm{~min}$. Slides were washed well with distilled water and left to air dry before being mounted by DPX (Sigma) and imaged using a Leica DM750 scope with Leica ICC50W digital camera attachment.

\section{$\underline{\text { FACS }}$}

Cells were countered using a haemocytometer (Marionfield) and dead cells were stained using trypan blue (sigma) diluted 1:10. Cells were disassociated and re-suspended in 10\% FCS (GIbco) 1xPBS and analysed using a BD LSRFORTESSA X-20 and dead cells were excluded using Hoechst's (Invitrogen), used at 1:10000. Cells were sorted using BD FACSAria $^{\text {TM }}$ Fusion. For $\gamma \mathrm{H} 2 \mathrm{AX}$, WKM cells were fixed in 4\% PFA for 10 mins at RT, permibilised in $0.1 \%$ Triton for 5 mins at RT, blocked in 4\% FBS for 20 mins at RT and stained with H2A.XS139ph (GeneTex, GTX127342) (diluted 1:1000 in 3\% FCS/PBS) followed by AlexaFluro 594 goat anti-rabbit (Invitrogen, A11012), (diluted 1:1000 in 3\% FCS/PBS). 
3'-scRNAseq was completed using Chromium Next GEM single cell 3' GEM library and Gel bead Kit v3.1 (10x Genomics) and sequenced using a NextSeq 500 (Illumina) at Genomics Birmingham (University of Birmingham). Analysis was completed using CellRanger, Loupe and R: Using Monocle 3 (Cao et al., 2019; Qiu et al., 2017a; Qiu et al., 2017b; Trapnell et al., 2014) and Seurat (Butler et al., 2018; Satija et al., 2015; Stuart et al., 2019). Results were presented using Graphpad Prism (v9). GO enrichment term analysis was completed biological function and using Metascape (Zhou et al., 2019).

\section{Immunofluorescence}

WKM cells were cytospun onto superfrost plus slide (Thermo Scientific) and left to air dry for $15 \mathrm{~min}$ at RT. Slides were pre-extracted (with pre-extraction buffer: $20 \mathrm{mM} \mathrm{NcCl}$, $3 \mathrm{mM} \mathrm{MgCl} 2,300 \mathrm{mM}$ sucrose, $10 \mathrm{mM}$ PIPES, $0.5 \%$ Triton $\mathrm{X}-100$ ) on ice for $5 \mathrm{~min}$ and then fixed in 4\% PFA for 10min at RT. Slides were washed in PBS 3 times and then blocked in 100ul blocking buffer (10\% FCS/PBS) for 1 hour at RT. H2A.XS139ph (GeneTex, GTX127342), 1:500 (diluted in 3\% FCS/PBS), was added to the slide for 1 hour at RT, washed in PBS and then incubated with AlexaFluro 594 goat anti-rabbit (Invitrogen, A11012), 1:1000 (diluted in 3\% FCS/PBS). Slides were then washed in PBS and mounted using DAPI mounting medium (abcam, ab104139).

\section{Cell culture and Western blot}

HPC7 murine multipotent progenitor cells were obtained from Prof J. Frampton (University of Birmingham), and maintained in StemPro-34 SFM (Invitrogen, 10639011) containing supplement, 1\% Pen/Strep, 1 x L-glutamine and $100 \mathrm{ng} / \mathrm{ml} \mathrm{SCF}$. Cells were treated with the NPM1 oligomerization inhibitor (Stratech Scientific Ltd) (Qi et al., 2008) 
(1 uM for $24 \mathrm{~h}$ ), harvested by centrifugation and whole cell extracts were obtained by lysis in UTB buffer (8 M Urea, $50 \mathrm{mM}$ Tris, $150 \mathrm{mM} \beta$-mercaptoethanol). Lysates were separated and analysed by SDS-PAGE following standard procedures. The following antibodies were used: $\gamma \mathrm{H} 2 \mathrm{AX}$ (Millipore; cat\#05-636; RRID:AB_309864), H2A (Millipore; cat\#07-146; RRID:AB_11212920), Anti-rabbit HRP (Agilent; cat\#P0399; RRID: AB_2617141), Anti-mouse HRP (Agilent; Cat\# P0447; RRID: AB_2617137).

\section{Acknowledgements}

We thank the staff of the Biomedical Services Unit for fish husbandry. We thank Dr Adriana Flores-Langarica and Dr Paola Pietroni from the flow cytometry facility (University of Birmingham) for cell sorting. We thank the staff of Genomics Birmingham (University of Birmingham) for genomic sequencing and Prof. J. Frampton for the HPC7 cells. This research was supported by a Welcome Trust ISSF Award to R.M. and by the British Heart Foundation (BHF IBSR Fellowship FS/13/50/30436 to R.M. and C.B.M.). 


\section{References}

Andrade, N.S., Ramic, M., Esanov, R., Liu, W., Rybin, M.J., Gaidosh, G., Abdallah, A., Del'Olio, S., Huff, T.C., Chee, N.T., et al. (2020). Dipeptide repeat proteins inhibit homology-directed DNA double strand break repair in C9ORF72 ALS/FTD. Molecular Neurodegeneration 15, 13.

Avagyan, S., Weber, M.C., Ma, S., Prasad, M., Mannherz, W.P., Yang, S., Buenrostro, J.D., and Zon, L.I. (2021). Single-cell ATAC-seq reveals GATA2-dependent priming defect in myeloid and a maturation bottleneck in lymphoid lineages. Blood Adv 5, 2673-2686.

Avellino, R., and Delwel, R. (2017). Expression and regulation of C/EBPa in normal myelopoiesis and in malignant transformation. Blood 129, 2083-2091.

Butler, A., Hoffman, P., Smibert, P., Papalexi, E., and Satija, R. (2018). Integrating single-cell transcriptomic data across different conditions, technologies, and species. Nature Biotechnology 36 , 411-420.

Cao, J., Spielmann, M., Qiu, X., Huang, X., Ibrahim, D.M., Hill, A.J., Zhang, F., Mundlos, S., Christiansen, L., Steemers, F.J., et al. (2019). The single-cell transcriptional landscape of mammalian organogenesis. Nature 566, 496-502.

Chi, N.C., Shaw, R.M., De Val, S., Kang, G., Jan, L.Y., Black, B.L., and Stainier, D.Y. (2008). Foxn4 directly regulates tbx2b expression and atrioventricular canal formation. Genes Dev 22, 734-739.

Colombo, E., Marine, J.C., Danovi, D., Falini, B., and Pelicci, P.G. (2002). Nucleophosmin regulates the stability and transcriptional activity of p53. Nat Cell Biol 4, 529-533.

Cooper, S., Guo, H., and Friedman, A.D. (2015). The +37 kb Cebpa Enhancer Is Critical for Cebpa Myeloid Gene Expression and Contains Functional Sites that Bind SCL, GATA2, C/EBP $\alpha$, PU.1, and Additional Ets Factors. PLoS One 10, e0126385.

Crane, G.M., Jeffery, E., and Morrison, S.J. (2017). Adult haematopoietic stem cell niches. Nature Reviews Immunology 17, 573-590.

Dai, Y., Zhu, L., Huang, Z., Zhou, M., Jin, W., Liu, W., Xu, M., Yu, T., Zhang, Y., Wen, Z., et al. (2016). Cebpo is essential for the embryonic myeloid progenitor and neutrophil maintenance in zebrafish. J Genet Genomics 43, 593-600.

de Pater, E., Kaimakis, P., Vink, C.S., Yokomizo, T., Yamada-Inagawa, T., van der Linden, R., Kartalaei, P.S., Camper, S.A., Speck, N., and Dzierzak, E. (2013). Gata2 is required for HSC generation and survival. J Exp Med 210, 2843-2850.

Dickinson, R.E., Milne, P., Jardine, L., Zandi, S., Swierczek, S.I., McGovern, N., Cookson, S., Ferozepurwalla, Z., Langridge, A., Pagan, S., et al. (2014). The evolution of cellular deficiency in GATA2 mutation. Blood 123, 863-874.

Dobrzycki, T., Mahony, C.B., Krecsmarik, M., Koyunlar, C., Rispoli, R., Peulen-Zink, J., Gussinklo, K., Fedlaoui, B., de Pater, E., Patient, R., et al. (2020). Deletion of a conserved Gata2 enhancer impairs haemogenic endothelium programming and adult Zebrafish haematopoiesis. Commun Biol 3, 71.

Ellett, F., Pase, L., Hayman, J.W., Andrianopoulos, A., and Lieschke, G.J. (2011). mpeg1 promoter transgenes direct macrophage-lineage expression in zebrafish. Blood 117, e49-56.

Falini, B., Mecucci, C., Tiacci, E., Alcalay, M., Rosati, R., Pasqualucci, L., La Starza, R., Diverio, D., Colombo, E., Santucci, A., et al. (2005). Cytoplasmic nucleophosmin in acute myelogenous leukemia with a normal karyotype. N Engl J Med 352, 254-266.

Ferrero, G., Gomez, E., Lyer, S., Rovira, M., Miserocchi, M., Langenau, D.M., Bertrand, J.Y., and Wittamer, V. (2020). The macrophage-expressed gene (mpeg) 1 identifies a subpopulation of B cells in the adult zebrafish. J Leukoc Biol 107, 431-443.

Gao, X., Johnson, K.D., Chang, Y.I., Boyer, M.E., Dewey, C.N., Zhang, J., and Bresnick, E.H. (2013). Gata2 cis-element is required for hematopoietic stem cell generation in the mammalian embryo. J Exp Med 210, 2833-2842.

Gioacchino, E., Koyunlar, C., Zink, J., de Looper, H., de Jong, M., Dobrzycki, T., Mahony, C.B., Hoogenboezem, R., Bosch, D., van Strien, P.M.H., et al. (2021). Essential role for Gata2 in modulating lineage output from hematopoietic stem cells in zebrafish. Blood Adv 5, 2687-2700. 
Grisendi, S., Bernardi, R., Rossi, M., Cheng, K., Khandker, L., Manova, K., and Pandolfi, P.P. (2005). Role of nucleophosmin in embryonic development and tumorigenesis. Nature 437, 147-153.

Hasemann, M.S., Lauridsen, F.K.B., Waage, J., Jakobsen, J.S., Frank, A.-K., Schuster, M.B., Rapin, N., Bagger, F.O., Hoppe, P.S., Schroeder, T., et al. (2014). C/EBPa Is Required for Long-Term Self-Renewal and Lineage Priming of Hematopoietic Stem Cells and for the Maintenance of Epigenetic Configurations in Multipotent Progenitors. PLOS Genetics 10, e1004079.

Healy, L., May, G., Gale, K., Grosveld, F., Greaves, M., and Enver, T. (1995). The stem cell antigen CD34 functions as a regulator of hemopoietic cell adhesion. Proc Natl Acad Sci U S A 92, 12240-12244.

Hirabayashi, S., Wlodarski, M.W., Kozyra, E., and Niemeyer, C.M. (2017). Heterogeneity of GATA2related myeloid neoplasms. Int J Hematol 106, 175-182.

Johnson, K.D., Hsu, A.P., Ryu, M.J., Wang, J., Gao, X., Boyer, M.E., Liu, Y., Lee, Y., Calvo, K.R., Keles, S., et al. (2012). Cis-element mutated in GATA2-dependent immunodeficiency governs hematopoiesis and vascular integrity. J Clin Invest 122, 3692-3704.

Kawamura, K., Qi, F., Meng, Q., Hayashi, I., and Kobayashi, J. (2019). Nucleolar protein nucleolin functions in replication stress-induced DNA damage responses. J Radiat Res 60, 281-288.

Kobayashi, I., Kobayashi-Sun, J., Hirakawa, Y., Ouchi, M., Yasuda, K., Kamei, H., Fukuhara, S., and Yamaguchi, M. (2020). Dual role of Jam3b in early hematopoietic and vascular development. Development 147.

Koike, A., Nishikawa, H., Wu, W., Okada, Y., Venkitaraman, A.R., and Ohta, T. (2010). Recruitment of phosphorylated NPM1 to sites of DNA damage through RNF8-dependent ubiquitin conjugates. Cancer Res 70, 6746-6756.

Kollárovič, G., Topping, C.E., Shaw, E.P., and Chambers, A.L. (2020). The human HELLS chromatin remodelling protein promotes end resection to facilitate homologous recombination and contributes to DSB repair within heterochromatin. Nucleic Acids Res 48, 1872-1885.

Lin, H.F., Traver, D., Zhu, H., Dooley, K., Paw, B.H., Zon, L.I., and Handin, R.I. (2005). Analysis of thrombocyte development in CD41-GFP transgenic zebrafish. Blood 106, 3803-3810.

Lindström, M.S. (2011). NPM1/B23: A Multifunctional Chaperone in Ribosome Biogenesis and Chromatin Remodeling. Biochem Res Int 2011, 195209.

Luissint, A.C., Williams, H.C., Kim, W., Flemming, S., Azcutia, V., Hilgarth, R.S., Leary, M.N.O., Denning, T.L., Nusrat, A., and Parkos, C.A. (2019). Macrophage-dependent neutrophil recruitment is impaired under conditions of increased intestinal permeability in JAM-A-deficient mice. Mucosal Immunol 12, 668-678.

Ma, D., Zhang, J., Lin, H.-f., Italiano, J., and Handin, R.I. (2011). The identification and characterization of zebrafish hematopoietic stem cells. Blood 118, 289-297.

McReynolds, L.J., Calvo, K.R., and Holland, S.M. (2018). Germline GATA2 Mutation and Bone Marrow Failure. Hematol Oncol Clin North Am 32, 713-728.

McReynolds, L.J., Yang, Y., Yuen Wong, H., Tang, J., Zhang, Y., Mule, M.P., Daub, J., Palmer, C., Foruraghi, L., Liu, Q., et al. (2019). MDS-associated mutations in germline GATA2 mutated patients with hematologic manifestations. Leuk Res 76, 70-75.

Moore, F.E., Garcia, E.G., Lobbardi, R., Jain, E., Tang, Q., Moore, J.C., Cortes, M., Molodtsov, A., Kasheta, M., Luo, C.C., et al. (2016). Single-cell transcriptional analysis of normal, aberrant, and malignant hematopoiesis in zebrafish. J Exp Med 213, 979-992.

Okuwaki, M. (2008). The structure and functions of NPM1/Nucleophsmin/B23, a multifunctional nucleolar acidic protein. J Biochem 143, 441-448.

Okuwaki, M., Matsumoto, K., Tsujimoto, M., and Nagata, K. (2001). Function of nucleophosmin/B23, a nucleolar acidic protein, as a histone chaperone. FEBS Lett 506, 272-276.

Peters, M.J., Parker, S.K., Grim, J., Allard, C.A.H., Levin, J., and Detrich, H.W., 3rd (2018). Divergent Hemogen genes of teleosts and mammals share conserved roles in erythropoiesis: analysis using transgenic and mutant zebrafish. Biol Open 7. 
Pinto do, O.P., Kolterud, A., and Carlsson, L. (1998). Expression of the LIM-homeobox gene LH2 generates immortalized steel factor-dependent multipotent hematopoietic precursors. Embo j 17, 5744-5756.

Qi, W., Shakalya, K., Stejskal, A., Goldman, A., Beeck, S., Cooke, L., and Mahadevan, D. (2008). NSC348884, a nucleophosmin inhibitor disrupts oligomer formation and induces apoptosis in human cancer cells. Oncogene 27, 4210-4220.

Qiu, X., Hill, A., Packer, J., Lin, D., Ma, Y.-A., and Trapnell, C. (2017a). Single-cell mRNA quantification and differential analysis with Census. Nature Methods 14, 309-315.

Qiu, X., Mao, Q., Tang, Y., Wang, L., Chawla, R., Pliner, H.A., and Trapnell, C. (2017b). Reversed graph embedding resolves complex single-cell trajectories. Nature Methods 14, 979-982.

Renshaw, S.A., Loynes, C.A., Trushell, D.M., Elworthy, S., Ingham, P.W., and Whyte, M.K. (2006). A transgenic zebrafish model of neutrophilic inflammation. Blood 108, 3976-3978.

Rodrigues, N.P., Boyd, A.S., Fugazza, C., May, G.E., Guo, Y., Tipping, A.J., Scadden, D.T., Vyas, P., and Enver, T. (2008). GATA-2 regulates granulocyte-macrophage progenitor cell function. Blood 112, 48624873.

Saida, S., Zhen, T., Kim, E., Yu, K., Lopez, G., McReynolds, L.J., and Liu, P.P. (2020). Gata2 deficiency delays leukemogenesis while contributing to aggressive leukemia phenotype in Cbfb-MYH11 knockin mice. Leukemia 34, 759-770.

Satija, R., Farrell, J.A., Gennert, D., Schier, A.F., and Regev, A. (2015). Spatial reconstruction of singlecell gene expression data. Nature Biotechnology 33, 495-502.

Sawai, C.M., Babovic, S., Upadhaya, S., Knapp, D., Lavin, Y., Lau, C.M., Goloborodko, A., Feng, J., Fujisaki, J., Ding, L., et al. (2016). Hematopoietic Stem Cells Are the Major Source of Multilineage Hematopoiesis in Adult Animals. Immunity 45, 597-609.

Scott, D.D., and Oeffinger, M. (2016). Nucleolin and nucleophosmin: nucleolar proteins with multiple functions in DNA repair. Biochem Cell Biol 94, 419-432.

Sharma, A., Singh, K., and Almasan, A. (2012). Histone H2AX phosphorylation: a marker for DNA damage. Methods Mol Biol 920, 613-626.

Shinya, M., Machiki, D., Henrich, T., Kubota, Y., Takisawa, H., and Mimura, S. (2014). Evolutionary diversification of MCM3 genes in Xenopus laevis and Danio rerio. Cell Cycle 13, 3271-3281.

Snyder, M., He, W., and Zhang, J.J. (2005). The DNA replication factor MCM5 is essential for Stat1mediated transcriptional activation. Proc Natl Acad Sci U S A 102, 14539-14544.

Soukup, A.A., Zheng, Y., Mehta, C., Wu, J., Liu, P., Cao, M., Hofmann, I., Zhou, Y., Zhang, J., Johnson, K.D., et al. (2019). Single-nucleotide human disease mutation inactivates a blood-regenerative GATA2 enhancer. Journal of Clinical Investigation.

Spinner, M.A., Sanchez, L.A., Hsu, A.P., Shaw, P.A., Zerbe, C.S., Calvo, K.R., Arthur, D.C., Gu, W., Gould, C.M., Brewer, C.C., et al. (2014). GATA2 deficiency: a protean disorder of hematopoiesis, lymphatics, and immunity. Blood 123, 809-821.

Sportoletti, P., Grisendi, S., Majid, S.M., Cheng, K., Clohessy, J.G., Viale, A., Teruya-Feldstein, J., and Pandolfi, P.P. (2008). Npm1 is a haploinsufficient suppressor of myeloid and lymphoid malignancies in the mouse. Blood 111, 3859-3862.

Stuart, T., Butler, A., Hoffman, P., Hafemeister, C., Papalexi, E., Mauck, W.M., III, Hao, Y., Stoeckius, M., Smibert, P., and Satija, R. (2019). Comprehensive Integration of Single-Cell Data. Cell 177, 18881902.e1821.

Suh, H.C., Gooya, J., Renn, K., Friedman, A.D., Johnson, P.F., and Keller, J.R. (2006). C/EBPalpha determines hematopoietic cell fate in multipotential progenitor cells by inhibiting erythroid differentiation and inducing myeloid differentiation. Blood 107, 4308-4316.

Tang, Q., Iyer, S., Lobbardi, R., Moore, J.C., Chen, H., Lareau, C., Hebert, C., Shaw, M.L., Neftel, C., Suva, M.L., et al. (2017). Dissecting hematopoietic and renal cell heterogeneity in adult zebrafish at singlecell resolution using RNA sequencing. J Exp Med 214, 2875-2887. 
bioRxiv preprint doi: https://doi org/10.1101/2021.07 19.452890; this version posted July 19, 2021. The copyright holder for this preprint (which was not certified by peer review) is the author/funder, who has granted bioRxiv a license to display the preprint in perpetuity. It is made available under aCC-BY-NC-ND 4.0 International license.

Trapnell, C., Cacchiarelli, D., Grimsby, J., Pokharel, P., Li, S., Morse, M., Lennon, N.J., Livak, K.J., Mikkelsen, T.S., and Rinn, J.L. (2014). The dynamics and regulators of cell fate decisions are revealed by pseudotemporal ordering of single cells. Nature Biotechnology 32, 381-386.

Traver, D., Paw, B.H., Poss, K.D., Penberthy, W.T., Lin, S., and Zon, L.I. (2003). Transplantation and in vivo imaging of multilineage engraftment in zebrafish bloodless mutants. Nature Immunology 4, 12381246.

Wang, X., Muramatsu, H., Okuno, Y., Sakaguchi, H., Yoshida, K., Kawashima, N., Xu, Y., Shiraishi, Y., Chiba, K., Tanaka, H., et al. (2015). GATA2 and secondary mutations in familial myelodysplastic syndromes and pediatric myeloid malignancies. Haematologica 100, e398-401.

West, R.R., Hsu, A.P., Holland, S.M., Cuellar-Rodriguez, J., and Hickstein, D.D. (2014). Acquired ASXL1 mutations are common in patients with inherited GATA2 mutations and correlate with myeloid transformation. Haematologica 99, 276-281.

Wlodarski, M.W., Collin, M., and Horwitz, M.S. (2017). GATA2 deficiency and related myeloid neoplasms. Semin Hematol 54, 81-86.

Yaqoob, N., Holotta, M., Prem, C., Kopp, R., and Schwerte, T. (2009). Ontogenetic development of erythropoiesis can be studied non-invasively in GATA-1:DsRed transgenic zebrafish. Comp Biochem Physiol A Mol Integr Physiol 154, 270-278.

Zhou, Y., Zhou, B., Pache, L., Chang, M., Khodabakhshi, A.H., Tanaseichuk, O., Benner, C., and Chanda, S.K. (2019). Metascape provides a biologist-oriented resource for the analysis of systems-level datasets. Nat Commun 10, 1523. 


\section{Figures}
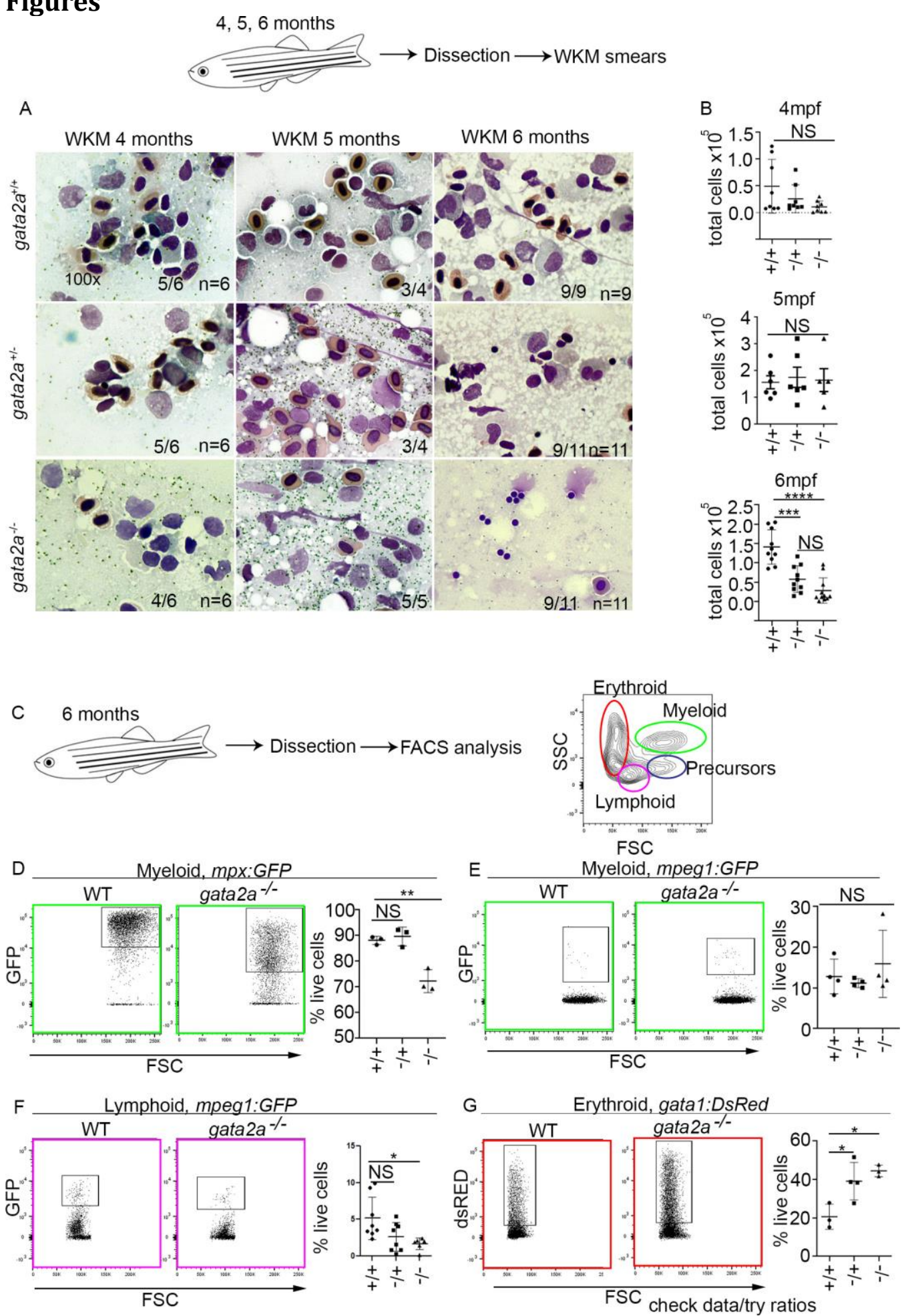

Figure 1. Loss of gata2a results in hypocellular marrow at $6 \mathbf{m p f}$

(A) WKM smears and May Grunewald/Giemsa sating at 4, 5 and 6mpf. (B) total WKM cell counts at 4, 5 and $6 \mathrm{mpf}$. (C) Flow cytometry gating strategy. (D-G) Analysis of different lineages using either mpx:GFP, mpeg1:GFP or gata1:DsRed transgenic lines. D, gata2 $\mathrm{a}^{+/+}$ vs gata $2 \mathrm{a}^{+/-:} \mathrm{p}=0.82$, gata $2 \mathrm{a}^{+/+}$vs gata $2 \mathrm{a}^{-/-}: \mathrm{p}=0.0024$. $\mathrm{E}$, gata $2 \mathrm{a}^{+/+}$vs gata $2 \mathrm{a}^{+/-:} \mathrm{p}=0.87$, gata $2 \mathrm{a}^{+/+}$vs gata $2 \mathrm{a}^{-/-}: \mathrm{p}=0.64 . \mathrm{F}$, gata $2 \mathrm{a}^{+/+}$vs gata $2 \mathrm{a}^{+/-}: \mathrm{p}=0.28$, gata $2 \mathrm{a}^{+/+}$vs gata $2 \mathrm{a}^{-/-}$: $\mathrm{p}=0.0239$. $\mathrm{G}$, gata $2 \mathrm{a}^{+/+}$vs gata $2 \mathrm{a}^{+/-}: \mathrm{p}=0.025$, gata $2 \mathrm{a}^{+/+}$vs gata $2 \mathrm{a}^{-/-}: \mathrm{p}=0.01$. Analysis was completed using one-way ANOVA, NS: $\mathrm{P}>0.05, *$ : $\mathrm{P}<0.05$. 
bioRxiv preprint doi: https://doi.org/10.1101/2021.07 19.452890; this version posted July 19, 2021. The copyright holder for this preprint (which was not certified by peer review) is the author/funder, who has granted bioRxiv a license to display the preprint in perpetuity. It is made available under aCC-BY-NC-ND 4.0 International license.

A
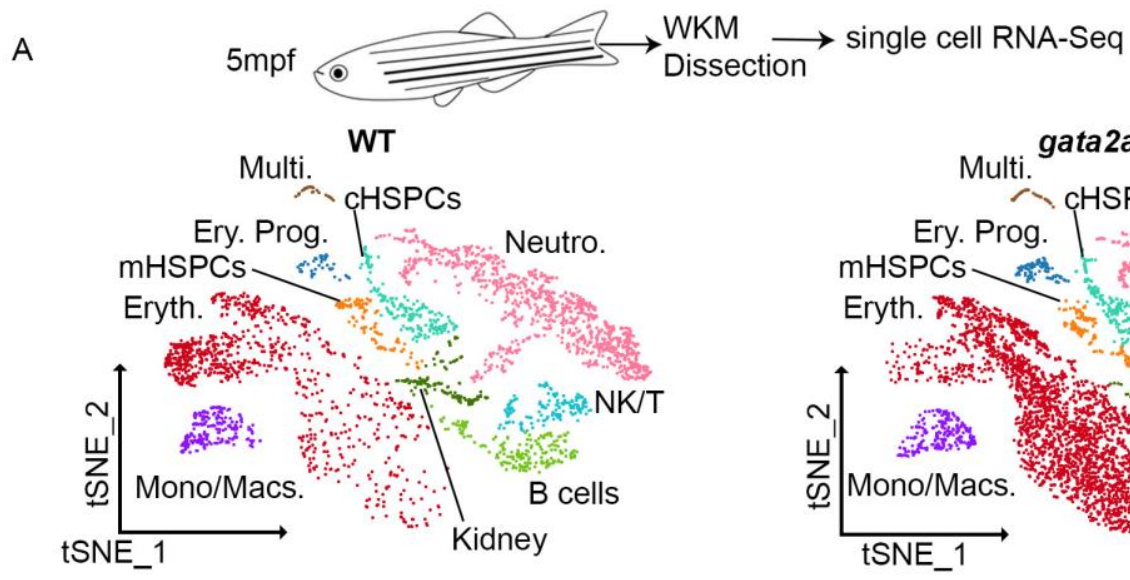

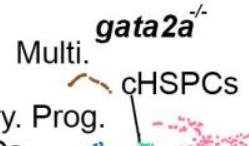
Ery. Prog. Neutro.

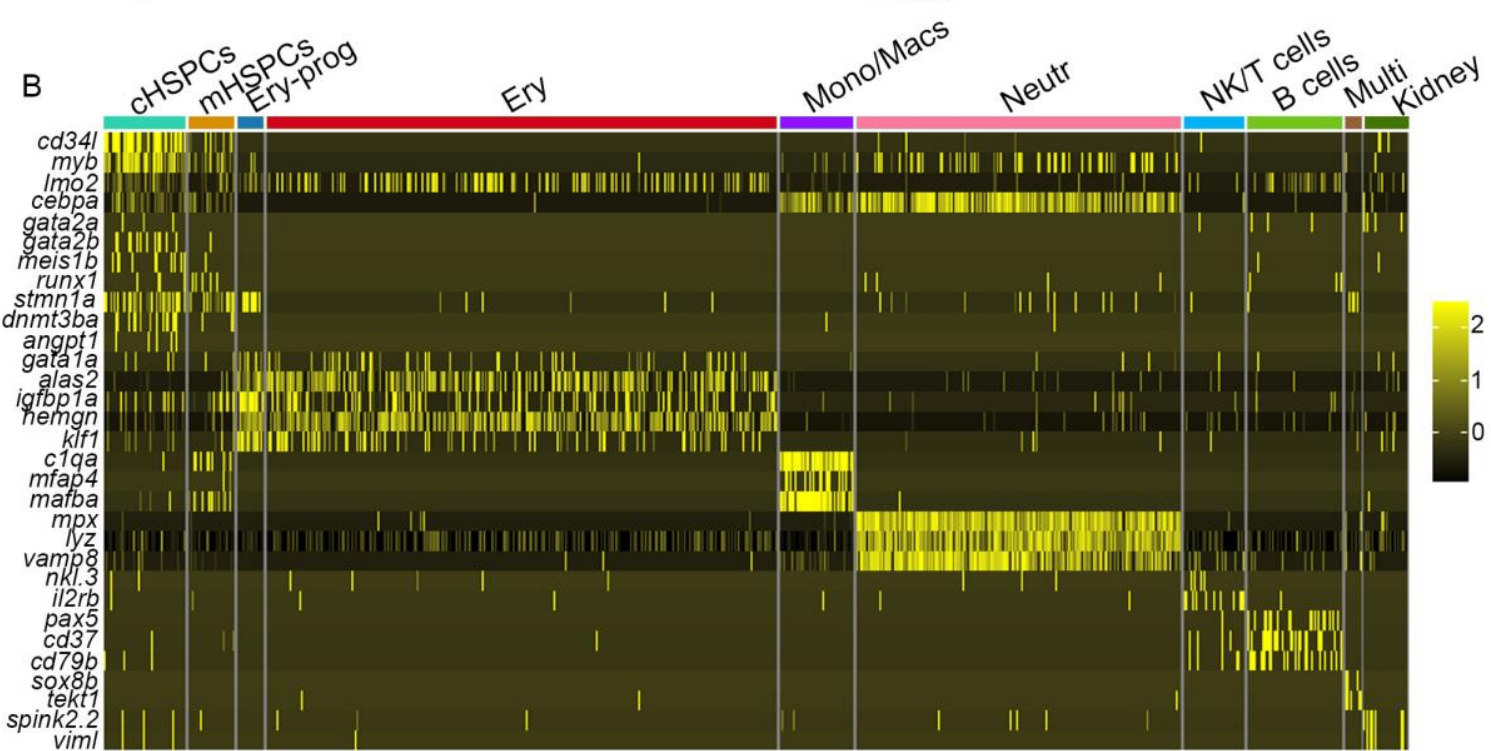

C $\%$ of total cells

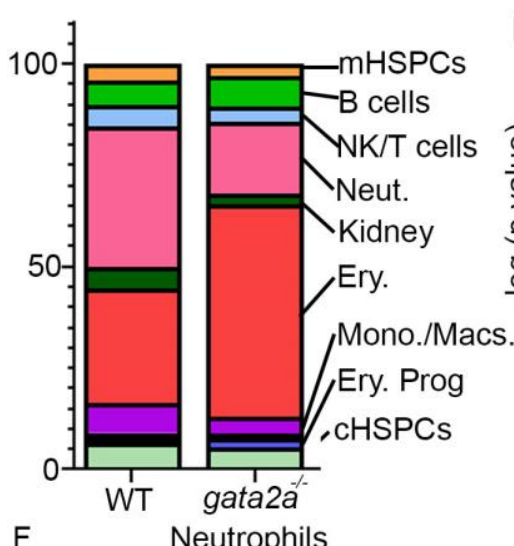

D Erythrocytes

lower in gata2a $a^{-/}$higher in gata2a $a^{-/-}$
$\mathrm{E}$

lower in gata2a $a^{-/}$higher in gata2a-

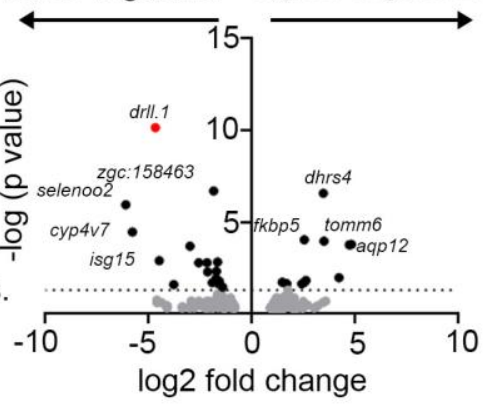

G

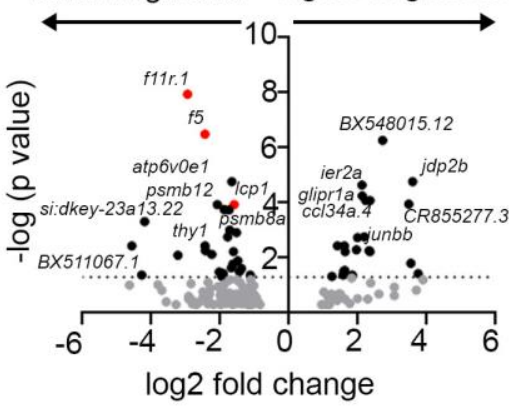

$\mathrm{H}$

$\mathrm{NK} / \mathrm{T}$ cells

B cells

lower in gata2a $a^{-/-}$higher in gata2a $a^{-/-}$lower in gata2a $a^{-/-}$higher in gata2a $a^{--}$lower in gata2a $a^{-/-}$higher in gata2a $a^{-/-}$
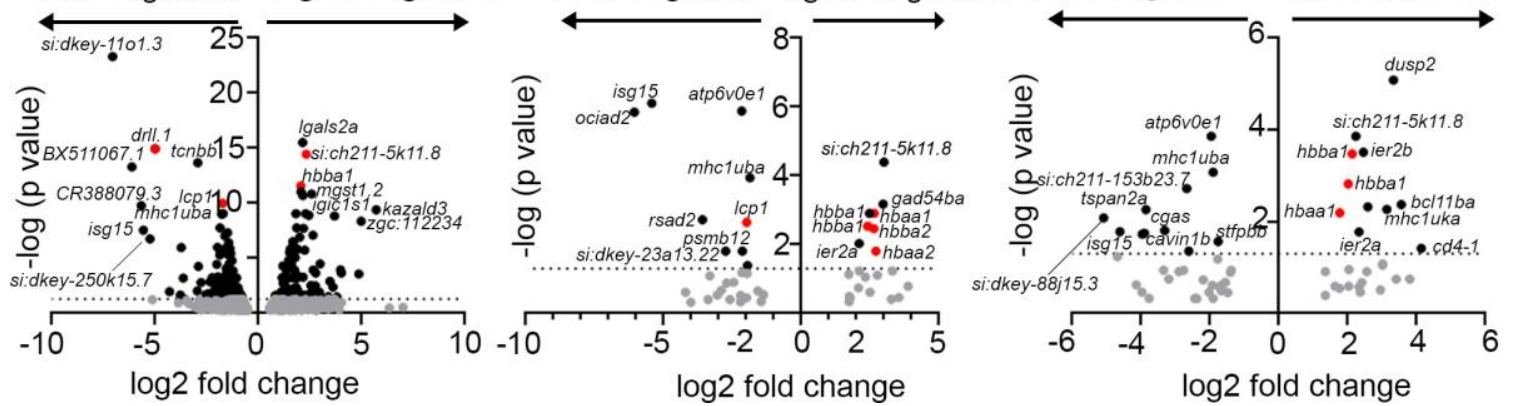
Figure 2. gata2a is required for lineage specification and transcriptional regulation in WKM haematopoietic populations

(A) scRNA-Seq and tSNE clustering of WKM haematopoietic populations. (B) Mean (Log2) expression levels of indicated genes in specific populations. cHSPCs: core HSPCs, mHSPCs: myeloid primed HSPCs, Ery Prog: erythroid progenitors, Mono/Macs.: Monocytes/Macrophages, Eryth: erythroid cells, Neut.: Neutrophils, NK/T: NK/T cells, Multi.: Multi ciliated cells. (C) Percentage of WKM populations of total WT or gata2a-/cells. (D-H) Volcano plots of log2 fold change vs -log10 ( $\mathrm{p}$ value, determined by using the Benjamini-Hochberg correction for multiple tests) in different WKM populations. Each dot represent a gene. Dotted line indicates $p=0.05$ with grey genes falling below this threshold. 
bioRxiv preprint doi: https://doi.org/101101/2021 07 19.452890; this version posted July 19, 2021. The copyright holder for this preprin (which was not certified by peer review) is the author/funder, who has granted bioRxiv a license to display the preprint in perpetuity. It is made available under aCC-BY-NC-ND 4.0 International license.

A

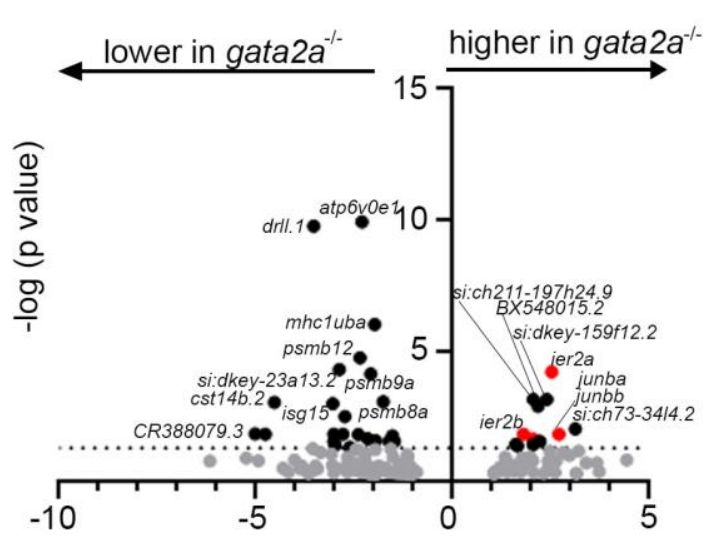

$\log 2$ fold change

C

cHSPC upregulated genes

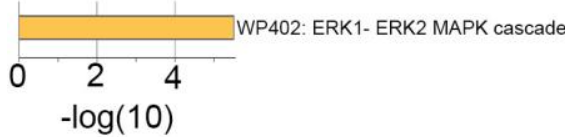

E

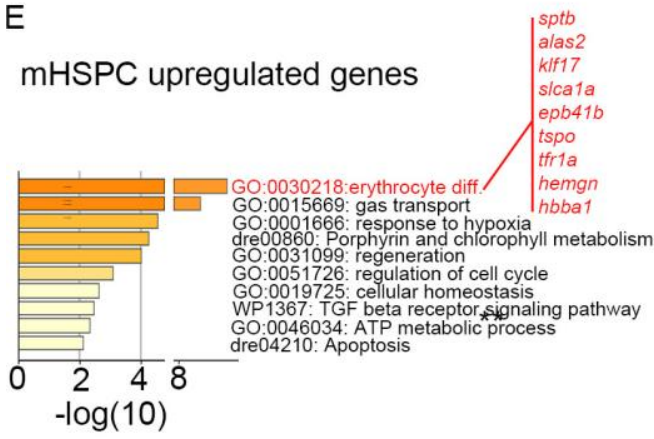

mHSPCS

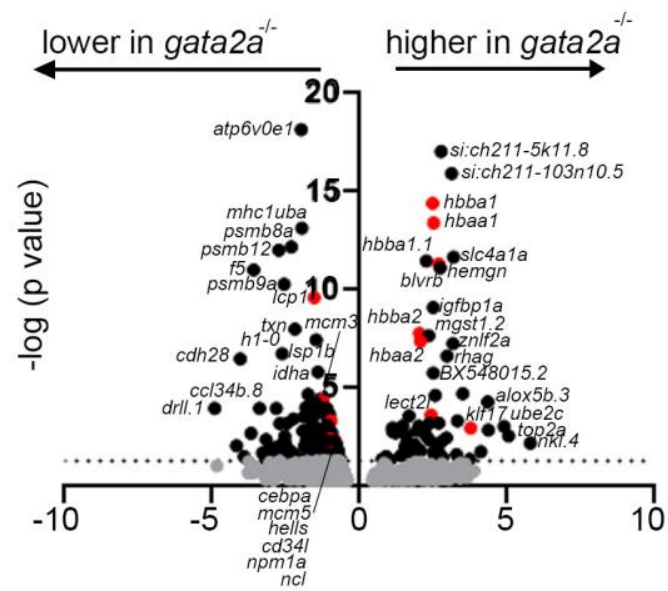

log2 fold change

D cHSPC downregulated genes

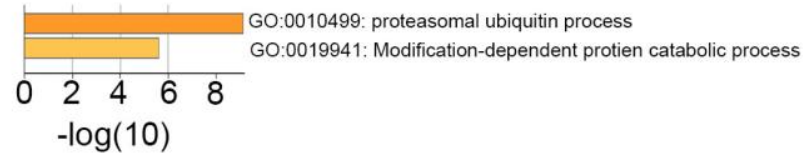

F

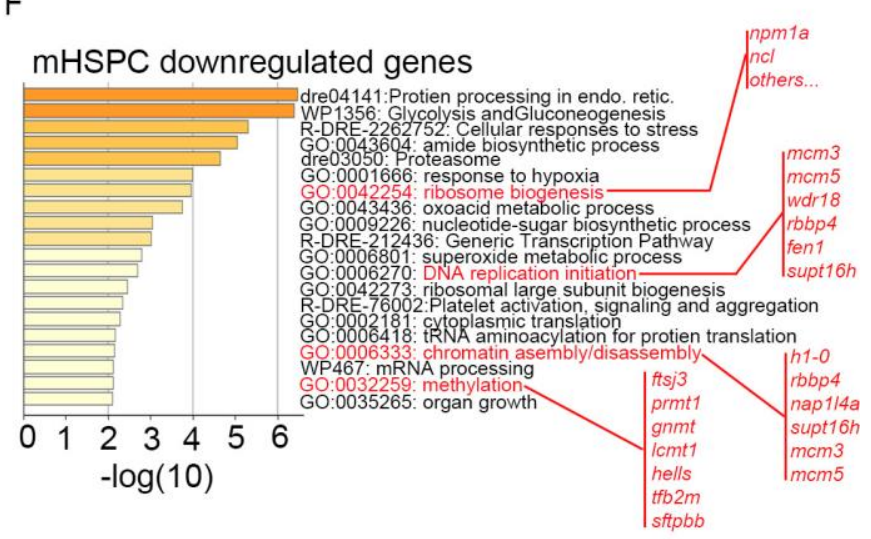

G
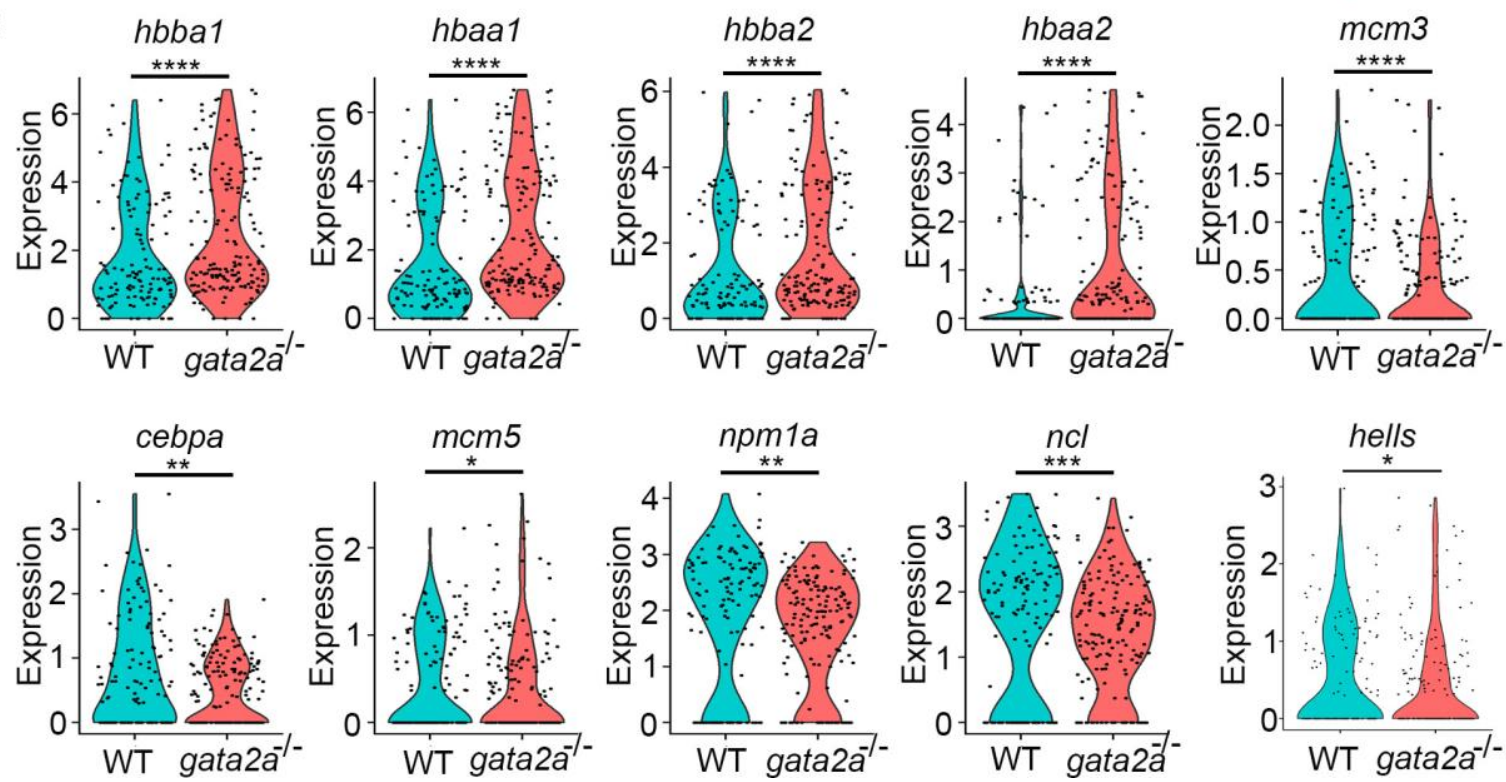
bioRxiv preprint doi: https://doi org/10.1101/2021.07 19.452890; this version posted July 19, 2021. The copyright holder for this preprint (which was not certified by peer review) is the author/funder, who has granted bioRxiv a license to display the preprint in perpetuity. It is made available under aCC-BY-NC-ND 4.0 International license.

Figure 3. Loss of gata2 in mHSPCs dysregulates the expression of differentiation and DNA repair genes.

(A, B) Volcano plots of $\log 2$ fold change vs $-\log 10$ ( $p$ value, determined by using the Benjamini-Hochberg correction for multiple tests) in cHSPCs (core HSPCs) and mHSPCs (Myeloid primed HSPCs) populations. Each dot represent a gene. Dotted line indicates $\mathrm{p}=0.05$ with grey genes falling below this threshold. (C-F) GO term enrichment (performed using metascape.org) analysis of up or down regulated genes in either cHSPCs or mHSPCs with a p value $<0.05$. (G) Expression levels were calculates using LogNormalize (Seurat: R package), by dividing feature counts by the total counts for that cell and then multiplied by the scale factor. This is then natural-log transformed using log1p. ${ }^{* * *}: \mathrm{p}<0.0001,{ }^{* * *}: \mathrm{p}<0.001^{* *}: \mathrm{p}<0.01,{ }^{*}: \mathrm{p}<0.05$ determined by using the Benjamini-Hochberg correction for multiple tests. 

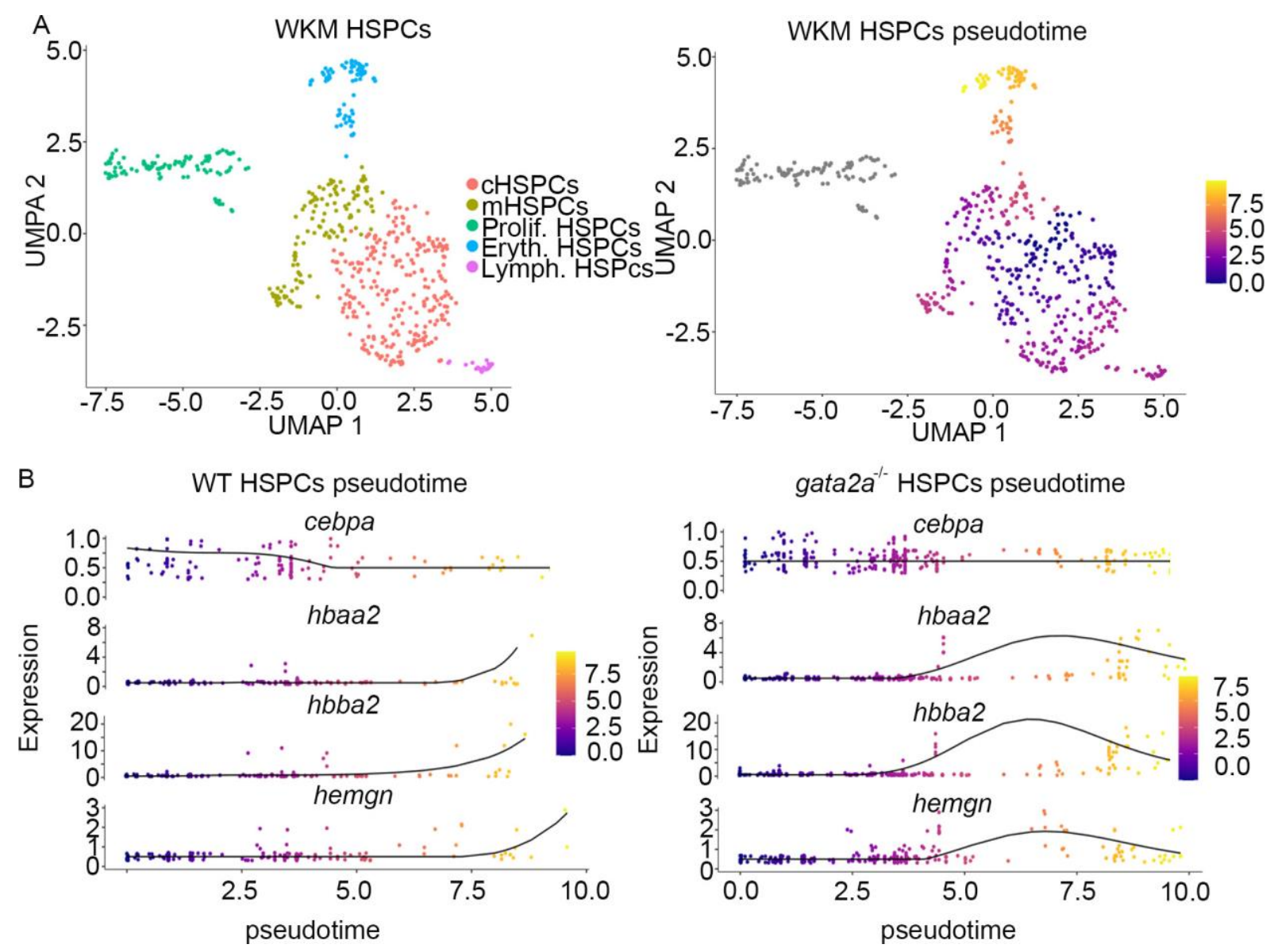

Figure 4. gata2a regulates cebpa expression as HSPCs differentiate

(A) UMAP plots of selected HSPCs clustered into different sub populations and ordered in pseudotime with cHSPCs defines as the point of differentiation origin. cHSPCs: core HSPCs, mHSPCs: myeloid primed HSPCs, Prolif. HSPCs: proliferating HSPCs, Eryth. HSPCs: erythroid primed HSPCs, Lymph. HSPCs: lymphoid primed HSPCs. (B) WT and gata2a/expression of cebpa, hbaa2, hbba2 and hemgn in pseudotime. 

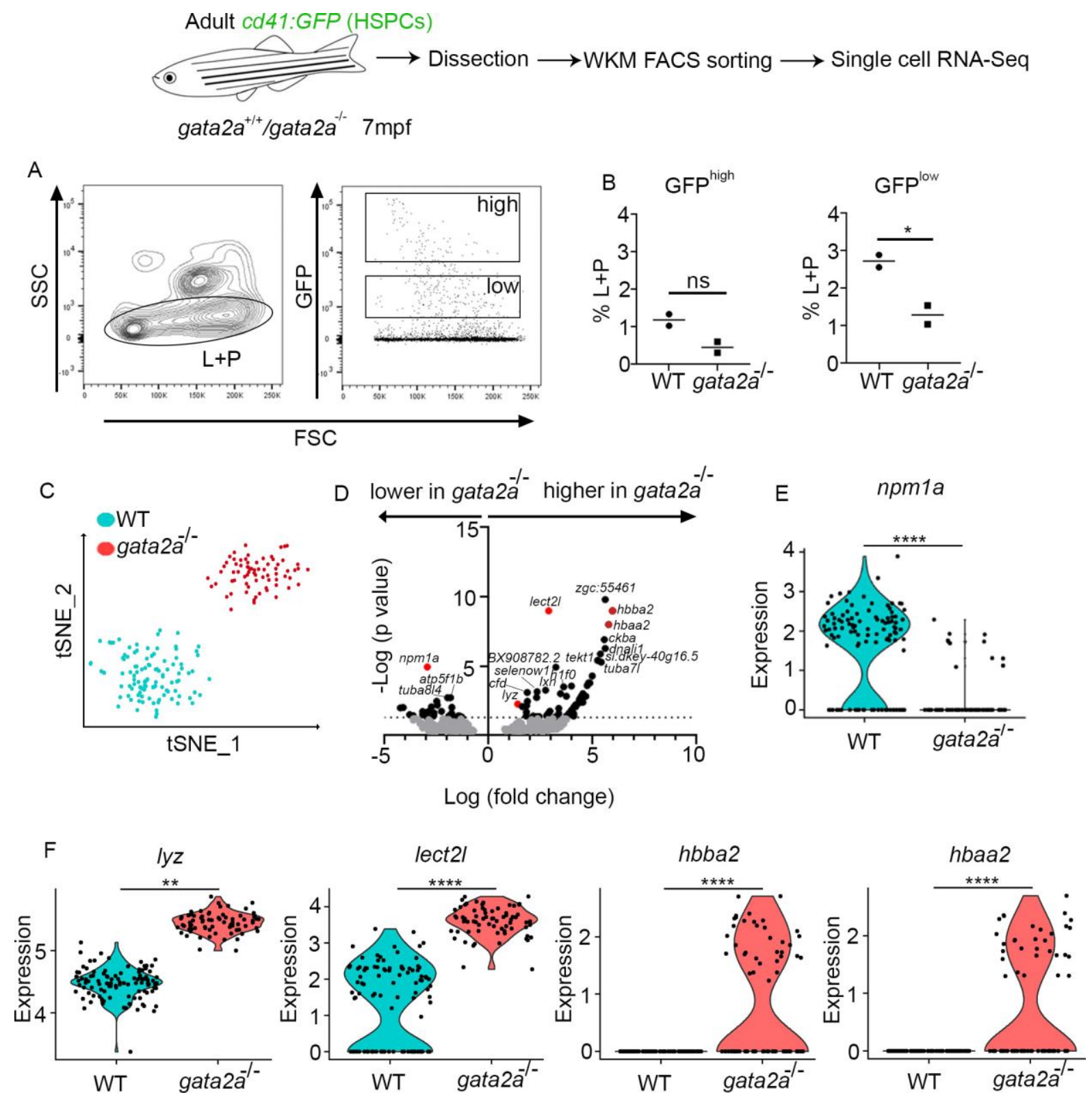

Figure 5. npm1a is down regulated as disease progresses in gata2a $/$ - adults.

(A) FACS gating strategy to analyse/isolate $c d 41$ :GFPlow from $7 \mathrm{mpf}$ adults. L: lymphoid cells, P: progenitor cells. (B) analysis of GFPhigh and GFPlow fraction. (C) scRNA-Seq and tSNE clustering of WT and gata2a $/$ cells. (D) Volcano plots of log2 fold change vs $\log 10$ (p value). (E) Violin plot (each dot represent a single cell) of npm1a expression in WT and gata $2 a^{-/-}$cells. (F) Violin plot (each dot represent a single cell) of lyz, lect2l, hbba2, and hbaa 2 expression in WT and gata $2 a^{-/}$cells. Expression levels were calculates using LogNormalize (Seurat: R package), by use dividing feature counts by the total counts for that cell and then multiplied by the scale factor. This is then natural-log transformed using log1p. ${ }^{* * * *}: \mathrm{p}<0.0001,{ }^{* *}: \mathrm{p}<0.01$, determined by using the Benjamini-Hochberg correction for multiple tests. 

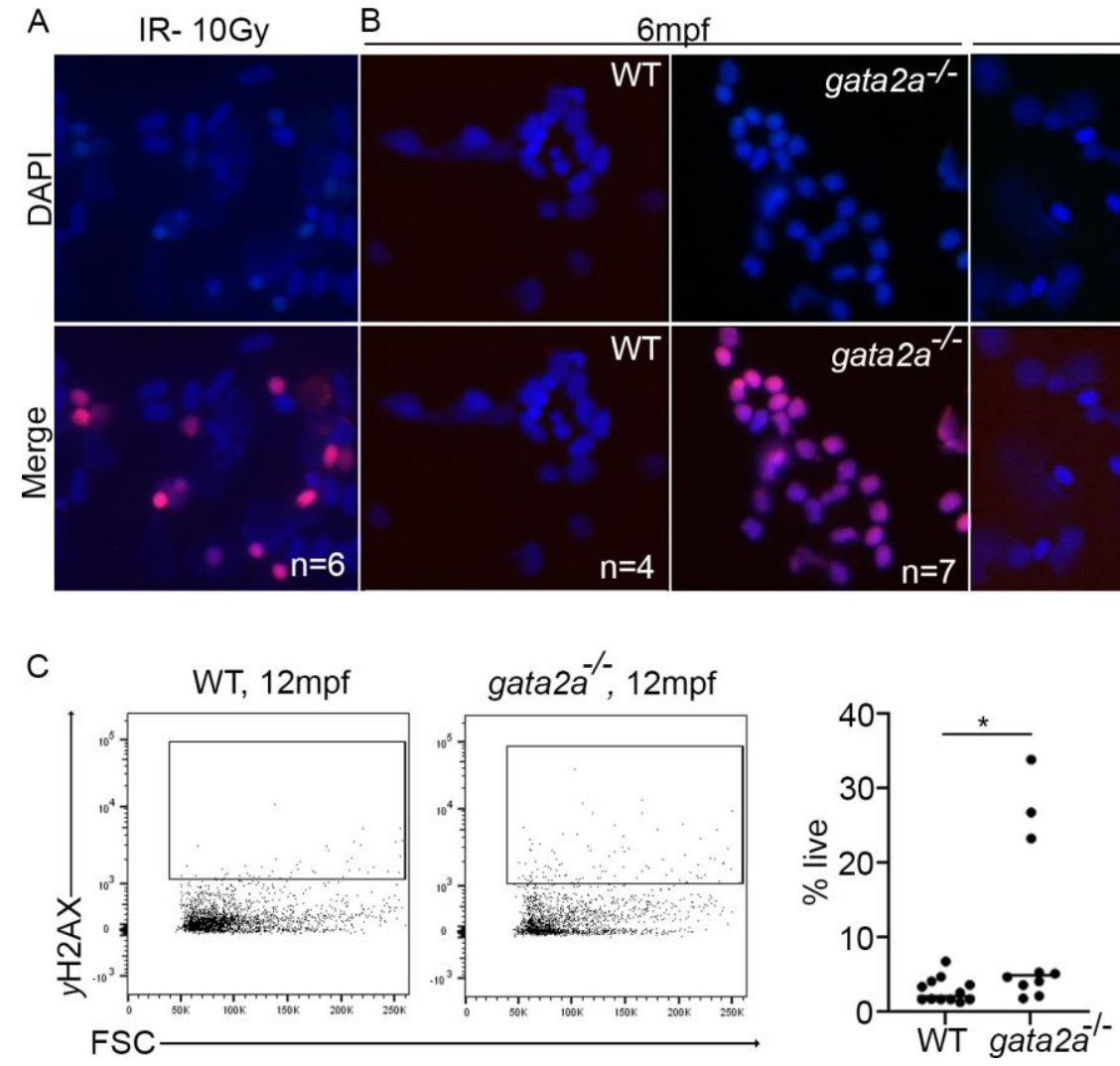

D

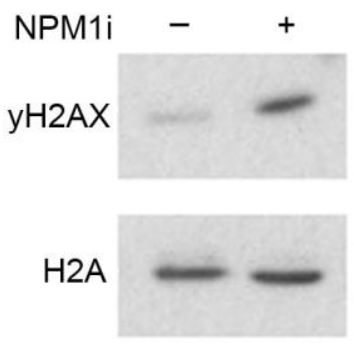

Figure 6. Loss of npm1a correlates with the accumulation of DNA damage.

(A) Control irradiation performed at $10 \mathrm{~Gy}$ on WKM cells and immunofluorescence against DAPI and $\gamma \mathrm{H} 2 \mathrm{AX}$ (B) WKM from $6 \mathrm{mpf}$ and $12 \mathrm{mpf}$ WT or gata2a $\mathrm{a}^{-}$adults and immunofluorescence against DAPI and $\gamma \mathrm{H} 2 \mathrm{AX}$. Imaging is at 40x magnification. (C) Flow cytometry analysis of $\gamma \mathrm{H} 2 \mathrm{AX}$ expression in $12 \mathrm{mpf}$ WKM suspension. $p=0.0296$. (D) Western blot of $\gamma \mathrm{H} 2 \mathrm{AX}$ and H2A expression in in HPC7 cells after $1 \mu \mathrm{M}$ NPM1 inhibitor for $24 \mathrm{~h} .{ }^{*}: p<0.05$, determined by using an unpaired, two tailed t-test. 